\title{
Surgical Management of Acquired Cervical Cystic Hygroma in a Nigerian Adult
}

\author{
OS Balogun, AA Osinowo, MO Afolayan, TO Olajide
}

General Surgery Unit, Department of Surgery, Faculty of Clinical Sciences, College of Medicine, University of Lagos \& Lagos University Teaching Hospital, PMB 12003, Idi-Araba. Lagos. Nigeria.

\begin{abstract}
Corresponding Author:
Dr Olanrewaju S Balogun

Email: drlanrebalogun@gmail.com licenses/by/3.0).

Received

April 24, 2017

Accepted

July 4, 2017

Published

July 20, 2017
\end{abstract}

This is an Open Access article distributed under the terms of the Creative Commons Attribution License (creativecommons.org/

\begin{abstract}
Background: Acquired cystic hygroma is unusual in adults and most of these lesions are found in the posterior triangle of the head and neck region. Onset in adults may be secondary to trauma, infection or blockage of lymphatics. Complete surgical excision is the treatment of choice in adults. Case Report: We report our encounter and surgical management of a female adult Nigerian with a long-standing acquired cervical cystic hygroma. The patient underwent complete surgical excision by transverse neck incision without any clinical recurrence or neurologic sequelae at follow-up. Conclusion: Total surgical excision is treatment of choice for acquired cystic hygroma.
\end{abstract}

Keywords: Cystic Lymphangioma, Edema, Humans, Lymphatic Vessels, Neck.

\section{Introduction}

Congenital abnormalities of the lymphatic system known as lymphangioma was first reported by Redenbacher in 1828 but the term "Cystic hygroma" was coined in 1843 by Wernher to describe the macro-cystic variety ( $>2 \mathrm{~cm}$ in size) of lymphangiomas [1]. Cystic hygroma is thought to result from congenital malformation of the lymphatic system occurring as early as $6^{\text {th }}$ week of intrauterine life. The adult-type of cystic hygroma usually presents as a soft one-sided asymptomatic neck lump which may be unilocular or multilocular. Although benign in nature, cystic hygroma has tendency for local infiltration of surrounding tissues making complete surgical excision difficult. We present our experience with surgical management of a 22 year old Nigerian woman with a large longstanding right-sided cystic hygroma of the neck.

\section{Case Report}

A 22 year old female student was referred to the surgical out-patient clinic of Lagos University
Teaching Hospital on account of painless right neck swelling of six years duration. The swelling was noticed two weeks after treatment for a chest infection. Initially it appeared like a soft small lemon-sized lump in the lower part of her neck but has gradually increased in size over the years to occupy the anterolateral side of her neck causing cosmetic concerns for the patient. She had no pressure symptoms on her aero-digestive tract. No night sweat, anorexia or weight loss was noted. Clinical examination revealed a well-nourished woman with a soft, fluctuant and non-tender mass involving the right side of her neck and ipsilateral jaw area. Her full blood count and erythrocyte sedimentation rates were unremarkable.

CT scan [Fig.1] revealed a large thin-walled cystic mass with enhancing septations measuring $92 \times 97 \times 116 \mathrm{~mm}$ in the right posterior triangle of the neck. There was displacement of trachea to the left. However, the fat planes between the mass and aero-digestive tract and the common carotid and internal jugular vein were preserved. Cytology of 
the aspirate from the swelling was negative for malignant cells. Patient had excision of right cervical cystic hygroma with insertion of suction drain under general anesthesia. Intra-operative findings included: a large multi-lobulated cystic swelling extending from the right jaw to the posterior triangle and the root of the neck with local extensions to deep trapezius and sternocleidomastoid muscles. We did not experience any anesthetic challenges peri-operatively. The patient was positioned in the left lateral position [Fig.2], skin preparation and draping was done. The cyst was approached via a transverse incision along the crease of the neck which has been marked with a thick silk suture. Local infiltration of the area of planned incision with one percent xylocaine with adrenaline significantly minimized bleeding on skin incision.

Sub-platysmal flaps were raised to expose the cyst wall. The cyst was found to be thin-walled [Fig.3] and attached to surrounding neck structures by loose and fibrous adhesions which yielded easily to sharp and blunt dissection. We experienced leakage of the cyst contents during our dissection and the cyst was further decompressed by gentle suction to facilitate its dissection from surrounding tissues. All delicate structures in the posterior triangle and adjoining areas were identified and preserved. Wound bed [Fig.4] was irrigated with saline, a gentle suction drain left in situ and wound closure in layers done with polyglactin sutures.

Immediate post-operative state of the patient was unremarkable. Suction drain was removed at 48 hours post-operatively and patient was discharged home at $4^{\text {th }}$ day post-surgery. Histology of excised specimen [Fig.5] revealed a benign lesion with dilated irregular vascular channel lined by flattened endothelium and surrounded by collagenous stroma confirming cystic type of lymphangioma. Multiple lymph nodes with reactive follicular hyperplasia were also seen. Her follow-up has shown a good cosmetic outcome with no clinical recurrence or neurologic sequelae [Fig.6].

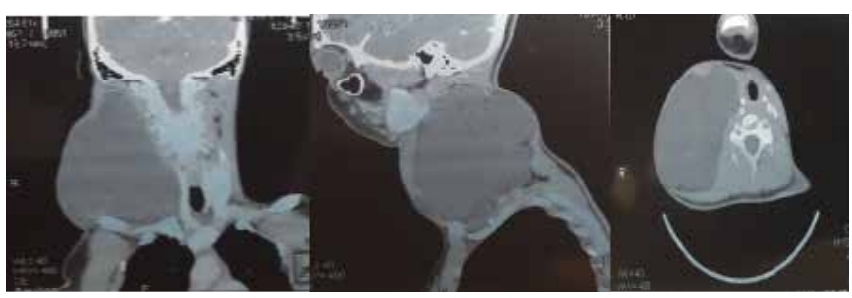

Fig.1: Coronal, sagittal and transverse head and neck CT scan showing a large cystic mass on right neck with displacement of the neurovascular bundles to the left side.



Fig.2: Patient position under general anesthesia.

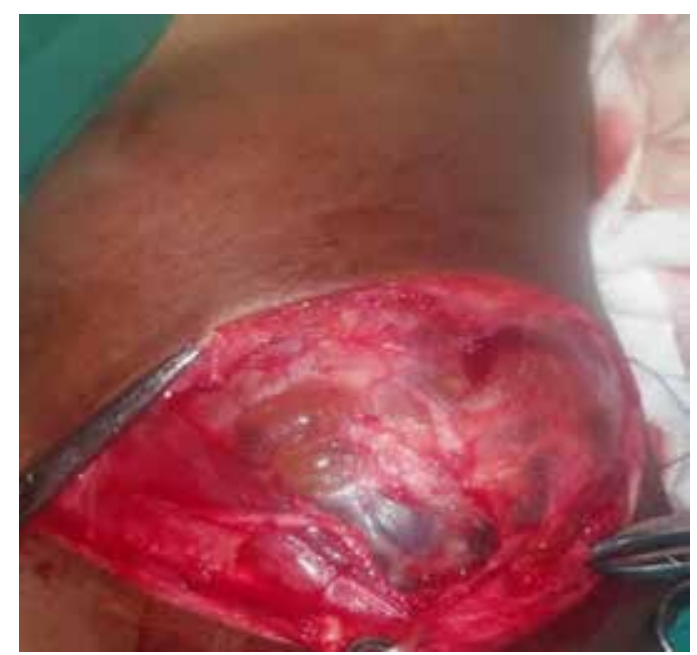

Fig.3: Transverse neck crease incisions showing thin cyst wall. 


\section{Discussion}

Acquired cystic hygroma is one of the unusual differential diagnosis of cystic neck lesions in adults. Trauma, infection and malignancies have been identified as precipitating factors. In our patient, this lesion was reported to have developed in the base of the posterior triangle of the neck following an episode of self-limiting respiratory tract infection. This was difficult to prove in our patient due to her late presentation to our clinic. Ho et al. reported a case of an adult-onset cystic hygroma in a 34 year old female from foreign body reaction to a retained wood in the neck following an episode of domestic violence [2]. However, etiology of most acquired cystic hygroma is uncertain even in documented cases of rapid development of symptoms $[3,4]$. The usual slowly progressive growth of this lesion coupled with absence of pressure symptoms on the aero-digestive tract and restriction of neck movements in adults may explain the reason for her late presentation to our clinic. Patient was only bothered by her cosmetic appearance.

Definitive management of cystic hygroma can be conservative, medical or surgical. There have been reports of spontaneous regression of fetal cystic hygroma [5]. This is however, an uncommon event in adults. Medical therapy using intra-lesional sclerosants such as ethanol, quinine, belomycin and OK-432 have been tried for the treatment of cystic hygroma but their use is limited by high recurrence rate, rapid increase in size of cystic hygroma with inflammation and alteration of normal tissue planes and lack of tissue sample for definitive histological diagnosis [2].

Surgical management is the mainstay of treatment of adult-onset cervical cystic hygroma and is based on the principle of meticulous and complete dissection of the cyst from adjacent tissues to prevent recurrence. Preservation of normal neurovascular structures of the neck is highly essential. Unlike congenital variety of cystic hygroma, the adult-type lesions are well defined



Fig.4: Wound bed after removal of the cyst.

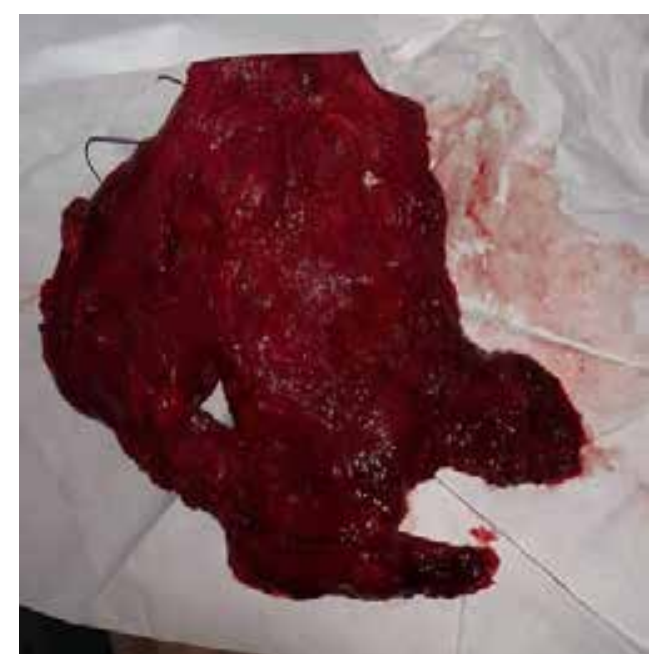

Fig. 5: Excised specimen.

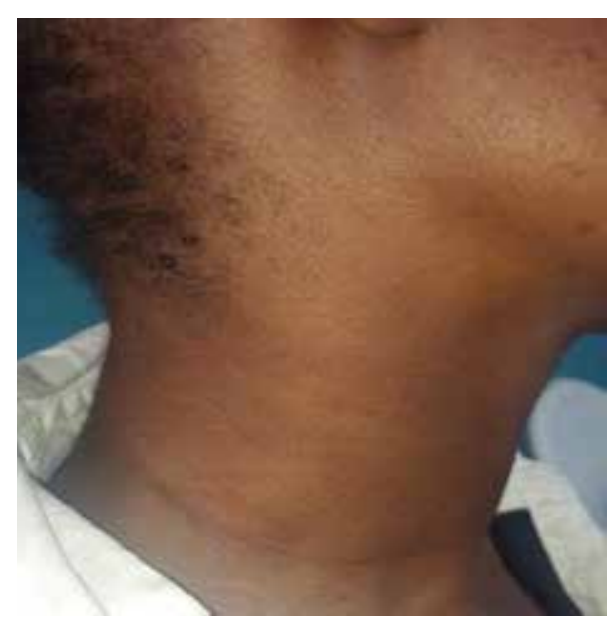

Fig.6: Patient at six months follow-up showing healed surgical scar. 
making complete surgical excision more feasible [6]. Complications of surgical excision include neuro-vascular injuries, intra-operative rupture of the cyst, wound sepsis and lymphatic discharge from the wound. Recurrence following surgical excision as reported by Kamath et al. may occur as early as four months after surgery necessitating a redo surgery [7]. A good pre-operative imaging showing well-defined tissue planes between the cyst and the vital structures of the neck are found to be predictors of complete surgical excision with low recurrence of cystic hygromas. Our findings on CT scan of the neck were found to correlate well with intra-operative findings in our patient. We were also able to completely excise the cyst without complications inspite of intraoperative cyst rupture that we encountered during the surgery. Our patient has been followed up till date without recurrence.

\section{Conclusion}

Acquired cystic hygroma in adults is a rare condition with variable presentation. The mainstay of treatment is surgery. Complete excision with preservation of the neurovascular bundle can be predicted with a good pre-operative imaging. Wellplaced transverse neck crease incision should be used where feasible for a good cosmetic outcome.
Contributors: OSB: manuscript writing, literature search and case management; AAO, MOA, TOO: manuscript editing, and literature search. OSB will act as guarantor. All authors approved the final version of the manuscript.

Funding: None; Competing interests: None stated.

\section{References}

1. Sherman BE, Kendall K. A unique case of large cystic hygroma in the adult. Am J Otolaryngol. 2001;22:206210.

2. Zhong PQ, Xhi FX, Li R, Xue JL, Shu GY. Long-term results of intratumorous bleomucin-A5 injection for head and neck lymphangioma. Oral Surg Oral Med Oral Pathol Oral Radiol Endodnone. 1998;86:139-144.

3. Gow L, Gulati R, Khan A, Mihaimeed F. Adult-onset cystic hygroma: A case report and review of management. Grand rounds. 2011;11:5-11.

4. Michail O, Michail P, Kyriaki D, Kolindou A, Klonaris C, Grimatsos J. Rapid development of an axillary cystic mass in an adult: A case of cystic hygroma. South Med J. 2007;100:845-849.

5. Watson WJ, Katz VL, Thorp JM. Spontaneous resolution of fetal nuchal cystic hygroma. J Perinatol. 1991;11:213215.

6. Naidu SI, McCalla MR. Lymphatic malformations of the head and neck in adults: a case report and review of the literature. Ann Otol Rhinol Laryngol. 2004;113:218-22.

7. Kamath B, Chatterjee A, Chandorkar I, Bhanushali H. Giant Recurrent Cystic Hygroma: A case report. J West African College of Surgeons. 2014;4:100-111. 